\title{
Lactobacillus rhamnosus/L. acidophilus/L. plantarum/ Enterococcus faecium Aqueous Probiotic Supplement
}

National Cancer Institute

\section{Source}

National Cancer Institute. Lactobacillus rhamnosus/L. acidophilus/L. plantarum/

Enterococcus faecium Aqueous Probiotic Supplement. NCI Thesaurus. Code C157490.

\begin{abstract}
A water-based multi-strain probiotic composed of an extract of germinated barley containing the four live, activated lactic acid bacteria strains Lactobacillus rhamnosus, L. acidophilus, L. plantarum, and Enterococcus faecium, with potential immunomodulatory and anti-inflammatory activities. Upon oral administration of Lactobacillus rhamnosus/L. acidophilus/L. plantarum/ Enterococcus faecium aqueous probiotic supplement, these four live bacteria modulate the composition of the normal gastrointestinal (GI) microflora, by increasing the beneficial bacteria and decreasing the harmful bacteria, and helps maintain adequate colonization of the Gl tract, thereby improving digestion and preventing Gl disturbances. These bacteria and the butyric acid produced by them create an environment unfavorable to pathogens by adhering to human epithelial cells, protecting epithelium by maintaining the tig ht junction integrity and forming a protective mucosal barrier. This prevents attachment of pathogens and reduces the risk of infection, and potentially reduces the severity of mucositis. By restoring gut microbiota, these bacteria may restore or enhance intestinal immune responses.
\end{abstract}

